\title{
A population-based algorithm for the multi travelling salesman problem
}

\author{
Rubén Iván Bolaños ${ }^{\mathrm{a}}$, Eliana $\mathrm{M}$. Toro $\mathbf{O}^{\mathrm{b}}$ and Mauricio Granada $\mathbf{E}^{\mathrm{a}^{*}}$
}

${ }^{a}$ Department of Electrical Engineering, Universidad Tecnológica de Pereira, Colombia

${ }^{b}$ Department of Industrial Engineering, Universidad Tecnológica de Pereira, Colombia

\begin{tabular}{l}
\hline C H R O N I C L E \\
\hline Article history: \\
Received July 222015 \\
Received in Revised Format \\
Septmber17 2015 \\
Accepted October 142015 \\
Available online \\
October 152015 \\
\hline Keywords: \\
Combinatorial optimization \\
Multi-traveling salesman problem \\
Population-based algorithm \\
Local search operators
\end{tabular}
\begin{abstract}
A B S T R A C T
This paper presents the implementation of an efficient modified genetic algorithm for solving the multi-traveling salesman problem (mTSP). The main characteristics of the method are the construction of an initial population of high quality and the implementation of several local search operators which are important in the efficient and effective exploration of promising regions of the solution space. Due to the combinatorial complexity of mTSP, the proposed methodology is especially applicable for real-world problems. The proposed algorithm was tested on a set of six benchmark instances, which have from 76 and 1002 cities to be visited. In all cases, the best known solution was improved. The results are also compared with other existing solutions procedure in the literature.
\end{abstract}

\section{Introduction}

The mTSP problem can be viewed from the perspective of two well-known problems: i) as a generalization of the Travelling Salesman Problem (TSP), where a set of routes is assigned to m salesmen who all start from and return to a home city, and ii) as a special case of the vehicle routing problem (VRP), in which customers are considered unitary demands and every travelling salesman only visits a predetermined number of cities. Thus, the mTSP can also be utilized for solving several types of VRPs and all formulations and solution approaches for the VRP are valid for the mTSP.

Although the VRP and TSP have been widely discussed in the literature, the research on the mTSP is limited. Moreover, few papers in the literature address the mTSP through efficient population-based algorithms. The main motivation for formulating a population methodology lies in the ease of integration with multi-objective strategies, which allow introducing practical aspects, such as profit, fuel consumption and environmental impact, among others. Therefore, it becomes relevant to develop and implement an effective and robust optimization methodology based on population. 
The main contributions of this paper are as follows: 1) An effective population algorithm is proposed; 2) different heuristic strategies that improve the quality of the initial population are presented; and 3) six local search operators are integrated into the methodology, with which a modified and improved version of the Genetic Algorithm presented by Chu and Beasley (1997) is obtained.

The obtained results demonstrate the efficiency of the improved method with respect to those reported in the literature.

The rest of the paper will proceed as follows. In Section 2, the literature of the mTSP is reviewed. In Section 3, the problem is defined, and the mathematical model is formulated. Section 4 describes the proposed algorithm. Results are reported in Section 5, and conclusions and perspectives are discussed in Section 6.

\section{Literature review}

While in specialized literature the work related to TSP and VRP are abundant and numerous instances and test systems are presented, relatively few studies are found on mTSP with which comparisons can be made regarding the best known solutions. In Bektas (2004), a complete review of the state of the art solutions that includes aspects of the mathematical formulation and solution procedures is presented. The author makes an interesting analysis of applications and connections with other problems, where the mTSP is associated with many applications such as, scheduling of print press (Gorenstein, 1970; Carter \& Ragsdale, 2002), bank crew (Svestka \& Huckfelt, 1973), interview (Gilbert \& Hofstra, 1992), photographer team (Zhang et al., 1999), security service (Calvo \& Cordone, 2003) and hot rolling (Tang et al., 2000), school bus routing (Angel et al., 1972), work force planning, workload balancing (Okonjo, 1988), mission planning for mobile robots can be found in Brumitt and Stentz (1998); Yu et al. (2002); Sariel et al. (2009) and inspection task-rescue scenarios, in which possible victims have to be identified (Faigl et al., 2005).

Likewise, several methodologies have been raised to solve the mTSP, such as heuristic and metaheuristic algorithms, neural network-based methods, ant systems and exact techniques. The mTSP is very time consuming due to its NP-hard nature. Therefore, heuristic algorithms are the preferred method. In this regard, Russell (1977) proposes a technique denominated as mTOUR heuristic that consists of two stages: cluster first-route second. In that work, the extended version of the Lin Kernighan algorithm (1973) is used for routing. Sze and Tiong (2007) perform a comparison between the Nearest Neighbor Algorithm (NNA) and Genetic Algorithm (GA) for solving the mTSP. The results obtained by the NNA are superior to the GA in terms of performance and computing time. However, a conventional GA was used in that case.

In a more recent work, Sedighpour et al. (2011) present an effective GA for solving the mTSP, in which the 2-Opt local search algorithm is used for improving solutions. In the aforementioned work, 6 benchmark instances from the TSPLIB (Reinelt, 2014) are used and, in all but four instances, the best known solution was improved.

Zhou and Li (2010) solve the mTSP problem by a modified GA. A greedy strategy is implemented to create the initial population, and the mutation operator is combined with the local search strategy 2-Opt, which allows one to quickly determine quality neighboring solutions and accelerates the convergence of the algorithm.

Chen et al. (2011) propose a genetic algorithm in which new mutation and recombination operators are suggested within a codification technique named two-part chromosome, representing the solution to the mTSP. This type of encoding takes into account the sequence and number of cities that must be visited by each traveling salesman. 
The work presented by Junjie and Dingwei (2006) applies an Ant Colony Optimization (ACO) to the mTSP with capacity constraints. The ACO tests several standard problems from TSPLIB, finding competitive solutions in reasonable computation times. However, for large instances, the algorithm does not reach the best known solution. Similarly, in Seidighpour et al. (2011), the results obtained by ACO are taken for comparison with those obtained in this paper.

A hybrid two-stage algorithm is presented by Yousefikhoshbakh and Seidighpour (2012); in the first stage, the mTSP is solved by the so-called sweep algorithm and in the second stage by so-called elite ant colony optimization in conjunction with a local search strategy 3-Opt to improve the solution found in the first stage. Six instances of the TSPLIB library are resolved showing the development and competitiveness of the algorithm.

Song et al. (2003) propose an extended simulated annealing, which is an open system, and this gives rise for perturbation schemes according to the problem-augmented TSP or mTSP. The entropy constraints are combined with an energy function to equally distribute the salesmen's workload. Further, that algorithm can solve the problem without any transformation into the standard form as proposed in Bellmore and Hong (1974) and GuoXing (1995).

Exact techniques have been explored to solve the problem. Initially, Laporte and Nobert (1980) solve mTSP without sub-tour elimination constraints. These restrictions are only included when an integer solution is found, whereby a labeling process is performed in order to verify if any subtour constraint has been violated. If a subtour is found in the solution, then the restrictions for each subtour are included in the problem, and a re-optimization is performed. The integrality is obtained using Gomory's cutting planes. The methodology is able to find optimal solutions for instances of size less than or equal to 100 cities.

\section{Problem definition and mathematical model}

The mTSP is NP-hard in the strong sense and is commonly formulated as an integer programming model that considers a complete directed graph $G(V, E)$ where $E$ is the set of edges and $V=\{0 \ldots n\}$ is the vertex set and corresponds to the cities; whereas vertex 0 corresponds to the depot. Graph $G$ must be strongly connected and is generally assumed to be complete. $D$ Is the matrix formed with the nonnegative metric cost $d_{i j}$ associated with each edge $(i, j) \in E$. The metric cost can represent cost, distance or time. $x_{i j}$ Is defined as a binary variable associated with each edge $(i, j) \in E$ that takes the value 1 if edge $(i, j)$ belongs to the optimal solution and takes the value 0 otherwise. In the mTSP, the cost matrix satisfies the triangle inequality, meaning $d_{i k}+d_{k j} \geq d_{i j} ; \quad \forall i, j, k \in V$. Therefore, given a set of cities where $m$ salesmen and a metric cost are located, the objective of the mTSP is to determine a tour for each salesman such that:

- The total tour cost is minimized.

- All of the routes must start and end at the depot.

- Each city must be visited exactly once by only one salesman.

Starting from a model based on a two-index vehicle flow formulation, the mTSP model may be defined as:

$$
\begin{aligned}
& \min \sum_{i \in V} \sum_{j \in V} d_{i j} x_{i j} \\
& \text { s.t } \\
& \sum_{i \in V} x_{i j}=1, \quad \forall j \in V \backslash\{0\}
\end{aligned}
$$




$$
\begin{aligned}
& \sum_{j \in V} x_{i j}=1, \quad \forall i \in V \backslash\{0\} \\
& \sum_{i \in V} x_{i 0}=m \\
& \sum_{j \in V} x_{0 j}=m \\
& \sum_{i \notin S} \sum_{j \in S} x_{i j} \geq r(S) \quad \forall S \subseteq V \backslash\{0\}, S \neq \varnothing \\
& x_{i j} \in\{0,1\} ; \quad \forall i, j \in E
\end{aligned}
$$

The indegree and outdegree Constraints (2) and (3) impose that exactly one edge enters and leaves the vertex associated with each city, respectively. Constraints (4) and (5) ensure that exactly $m$ salesmen leave from and return to the depot. The so-called capacity-cut constraints (6) impose both the connectivity of the solution and the maximum number of nodes that can be visited by any salesman. In fact, they indicate that each cut $(V \backslash S, S)$ defined by a customer set $s$ is crossed by a number of edges not smaller than $r(s)$ (minimum number of salesmen needed to serve set $s$ ). Alternatively, a family of equivalent constraints may be obtained by considering the subtour elimination constraints proposed for the TSP by Miller et al. (1960).

$$
u_{i}-u_{j}+p x_{i j} \leq p-1 \quad \forall i, j \in V \backslash\{0\}, i \neq j
$$

where $u_{i} \in V \backslash 0$, is an additional continuous variable representing the number of cities that salesman $i$ has not visited yet and $p$ is the maximum number of nodes that can be visited by any salesman. The above mathematical model (2) - (7) can be implemented in commercial solvers to obtain exact solutions of the mTSP only for small-sized instances (approximately less than 100 cities). Given $n$ is the number of cities to be visited and $m$ the total number of salesmen, the total number of possible routes covering all cities is given by $m(n-1) ! / 2$. Due to its high computational complexity, solving even moderate-sized mTSPs takes unreasonable computational time. The approximate approach never guarantees an optimal solution but provides a near optimal solution in an acceptable computational time.

\section{Methodology: Modified Chu-Beasley Genetic Algorithm (MCBGA)}

In this section, a modified genetic algorithm based on the Chu and Beasley (1997) approaches is proposed. The first part of the MCBGA requires a completely diverse initial population where every chromosome is unique. A tournament selection is performed to choose two parent solutions from the initial population. The chosen two are used for a special crossover adapted to the mTSP. The resulting child solutions from the crossover operator are converted into individual routes. The objective function is evaluated for each chromosome, and the one with the best score is chosen to undergo a mutation process. The mutation process is different from the traditional mutation. This is an improvement step. This stage includes some inter and intra-route heuristics. Finally, a new individual enters into the population. The process is performed iteratively until a convergence point. Each of these steps is presented in detail in the following sub-sections:

\subsection{Codification}

Let $n$ be the number of cities to be visited and $m$ the number of traveling salesmen available to visit all cities. Let $C=\left\{c_{1}, c_{2}, \ldots, c_{i}, \ldots, c_{m}\right\}$ be a set of size $m$, where $c_{i}$ is the number of cities contained in cluster TSP $P_{i}$. Thus, $T S P_{i}$ is a permuted set of cities that have to be connected through a feasible route for the traveling salesman $i$ such that $T S P_{i} \subseteq V \backslash 0$. In general, the representation of a feasible solution $\Pi$ to the mTSP is 
encoded by a chromosome that contains all cities to be visited (n-th order permutation), as shown in Expression (9).

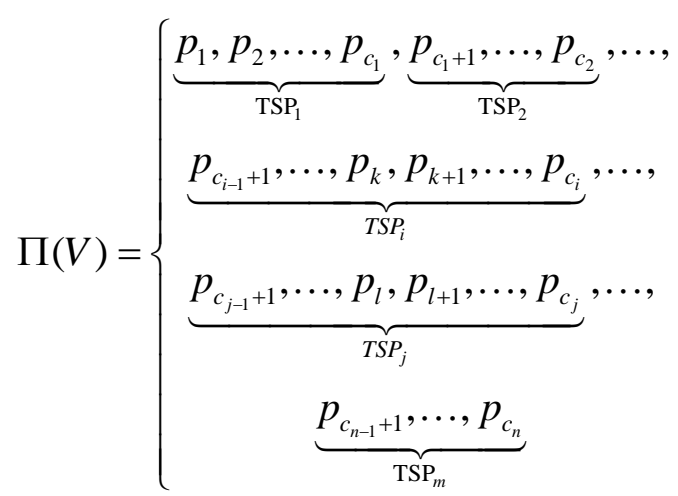

The number of cities in a cluster $i, c_{i}=\left|T S P_{i}\right|$, has a lower limit $c_{\text {min }}$ and an upper limit $c_{\text {max }}$, as shown in Eq. (10).

$$
c_{\min } \leq\left|T S P_{i}\right| \leq c_{\text {max }}
$$

The limit $c_{\text {max }}$ is an input parameter of the problem, while the limit $c_{\text {min }}$, which represents the minimum number of cities that each salesman must visit, is calculated by the following equation:

$$
c_{\text {min }}=\left\lfloor\frac{n}{T S P_{\text {min }}+1}\right\rfloor,
$$

where $T S P_{\min }$ represent the minimum number of salesmen needed to cover all cities and is calculated as:

$$
T S P_{\min }=\left\lceil\frac{n}{c_{\max }}\right\rceil
$$

\subsection{Initial population}

The initial population is obtained from $N$ approximated solutions of the traditional TSP considering the following: a) the complete set of cities except the deposit and b) a different starting city in each solution process. This strategy allows $N$ sequences or permutations of cities called Giant Tours. Using this sequence as a starting point, the generation of high quality individuals is achieved, which can be explained by the fact of being incorporated within each solution $\Pi$ several arcs for which the objective function is highly sensitive. The steps to build the individuals of the initial population are as follows:

- Each giant tour is built considering three heuristics: the nearest neighbor (Gutin et al., 2002), the heuristic methods for TSP of Christofides (1976) and Lin and Kernighan (1973). Initially, a percentage of the population (30\% in this case) is formed with the sequences obtained by the nearest neighbor method considering different starting cities. Another percentage of the population (30\% in this case) is generated using Christofides heuristics involving different starting cities on the Eulerian graph. It should be noted that this process begins with the construction of a Minimum Spanning Tree (MST) on the complete graph $G(V, E)$. From an Eulerian graph, the MST is constructed by pairing the vertices of odd degree of the MST, and, as a final step, a Hamiltonian graph is constructed from the Eulerian graph. Finally, the remaining percentage of the population is obtained from a solution resulting by applying LKH. This solution is slightly modified through exchanges between genes in order to obtain the remaining individuals 
of the population. It should ensure a diverse population.

- The giant tour is divided into $m$ clusters, where the number of cities of each cluster is chosen randomly, ensuring compliance with Constraint (10) resulting in $m-1$ routes. The route $m$ is formed by the cities that have yet to be allocated according the order established on each chromosome.

- Each chromosome is evaluated according to Equation (1) in order to obtain the initial value of the objective function.

\subsection{Selection and crossover}

Tournament-selection is used to select individuals who will be submitted to crossover under a given probability $\rho_{c}$. Goldberg and Deb (2004) indicate that tournament selection has the best convergence properties and computational complexity when compared with other selection operators presented in the literature.

Being a permutation-based encoding, the application of the crossover operator requires some care. A constraint of the problem is that all cities are visited only once. This implies that two genes on the chromosome cannot have the same value. Different strategies can be applied to prevent infeasible sequences, such as Partially Mapped Crossover (PMX) (Goldberg \& Lingle, 1985), Order Crossover (OX) (Oliver et al., 1987), Order Crossover \#2 (OX2) (Syswerda, 1991), Position Based Crossover (PBX) (Syswerda, 1991) and Cycle Crossover (CX) (Oliver et al.,1987). In this paper, the PMX method is used.

\subsection{Local-search}

The offspring obtained so far is subjected to mutation under a given probability_m. The mutation process is based on the application of neighborhood structures seeking to improve each of the routes obtained after application of the crossover operator. The upgrading steps applied are neighborhood structures known as inter-routes and intra-routes.

\subsubsection{Inter-route structures}

Five neighborhood structures involving inter-routes movements are applied (Subramanian et al. 2012), each of which is described below:

- Shift $(1 ; \mathbf{0})$, city $\mathrm{pk}_{\mathrm{k}}$ is transferred from route TSPi to route $\mathrm{TSP}_{\mathrm{j}}$.

- Shift $(2 ; 0)$, two adjacent cities $\mathrm{p}_{\mathrm{k}}$ and $\mathrm{pk}_{\mathrm{k}+1}$, are transferred from route TSP $\mathrm{P}_{\mathrm{i}}$ to route $\operatorname{TSP}_{\mathrm{j}}$.

- Swap (1; 1), a permutation is performed between city pk from route TSPi and city pı from route $\mathrm{TSP}_{\mathrm{j}}$.

- Swap (2; 1), two adjacent cities $\mathrm{p}_{\mathrm{k}}$ and $\mathrm{p}_{\mathrm{k}+1}$ from route $\mathrm{TSP} \mathrm{P}_{\mathrm{i}}$ are permuted with city $\mathrm{p}_{\mathrm{l}}$ from route $\mathrm{TSP}_{\mathrm{j}}$.

- Swap (2; 2), two adjacent cities $\mathrm{p}_{\mathrm{k}}$ and $\mathrm{p}_{\mathrm{k}+1}$ from route $\mathrm{TSP}_{\mathrm{i}}$ are permuted with two adjacent cities $\mathrm{pl}$ and $\mathrm{pl}+1$ from route $\mathrm{TSP}_{\mathrm{j}}$.

\subsubsection{Intra-route structure}

The local search algorithm 2-Opt is used as the intra-route neighborhood structure, which consists of removing two nonadjacent edges $E\left(\mathrm{pk}_{\mathrm{k}} ; \mathrm{p}_{\mathrm{k}+1}\right)$ and $E\left(\mathrm{pl} ; \mathrm{pl}_{\mathrm{l}+1}\right)$ belonging to the same route $\mathrm{TSP} \mathrm{P}_{\mathrm{i}}$, and then two arcs $E^{\prime}\left(\mathrm{p}_{\mathrm{k}} ; \mathrm{p}_{\mathrm{k}+1}\right)$ and $E^{\prime}(\mathrm{pl} ; \mathrm{pl}+1)$ are added so that a new route TSP $^{\prime}{ }_{\mathrm{i}}$ is created. 
Inter and intra-route neighborhood structures are applied in the order in which they were described and applied exhaustively, in order to choose the feasible movement to improve to a greater extent the value of the objective function and thus accelerate convergence to quality solutions.

\subsection{Population substitution}

When a new individual is obtained by the genetic operators of selection, recombination and mutation, an evaluation is necessary to determine whether that individual can be part of the population. The steps for population substitution are as follows:

- Verify that the objective function value of the new individual is less than the value of the objective function of the worst individual in the current population.

- Verify that the new individual does not exist in the current population.

If the two conditions described above are met, then the new individual enters the population replacing the individual of lower quality. The general methodology structure is presented in Algorithm 1.

Data: Instance size $\mathrm{n}$, population size $\mathrm{N}$, probability $\rho_{m}$ and $\rho_{c}$, maximum cities per route $c_{\text {max }}, \mathrm{P} \leftarrow\{\}$.

Result: Feasible optimal solution $\Pi$ to the the mTSP

while $|P| \neq n$ do

$\mathrm{P} \leftarrow \mathrm{P} \cup$ chromosomes from nearest neighbor, Christo_des algorithm and Lin-

Kernighan heuristic;

end

Calculate the minimum of routes TSPmin according to (12);

Calculate the minimum of cities per route cmin according to (11);

for $i \leftarrow 1 . . N$ do

Randomly split each chromosome with route size between $c_{\min }$ and $c_{\max }$;

end

while - stopping criteria (the fitness of the best solution has converged or a maximum number of generations has been reached ) do

$$
\begin{aligned}
& \left.p_{1}, p_{2} \leftarrow \text { tournament-selection ( } \mathrm{P}\right) \text {; } \\
& \text { if }\left(p_{1} \neq p_{2}\right) \text { and }\left(\operatorname{rand}() \leq \rho_{c}\right) \text { then } \\
& h \leftarrow \operatorname{Crossover}\left(p_{1}, p_{2}\right) \text {; } \\
& h \leftarrow p_{1} ;
\end{aligned}
$$

end

return The best solution $(P)$

Algorithm 1. MCBGA Pseudo code 


\section{Computational results}

In order to demonstrate the effectiveness of the proposed methodology tests on six standard instances of the library TSPLIB (Reinelt, 2014) are carried out, and the results obtained are compared to the results obtained by the algorithms ACO (Junjie \& Dingwei, 2006) and MGA (Seidighpour et al. 2011). The instances used in the tests are referred to as pr76, pr152, pr226, pr299, pr436 and pr1002.

The proposed algorithm is implemented in the programming environment Matlab 2010a on a PC Core 2 Duo with 3 GHz, 4 GB RAM Memory and Windows 7.

The MCBGA is characterized by an intensive neighborhood search. For this reason, the tests were conducted with a mutation rate of $\rho_{\mathrm{m}}=0.9$. The crossover rate used was $\rho_{\mathrm{c}}=1.0$.

Table 1 shows a comparison between the results obtained by algorithm MCBGA and ACO and MGA algorithms. The best solution found for each instance and the average value of the objective function after 10 runs of the algorithm are reported. The amount of travel agents used (m); the maximum number of cities (cmax) and the total number of cities to visit (n) are also reported.

The proposed algorithm finds better solutions for six instances used in this work with respect to the results reported in the literature for the same six instances (Seidighpour et al., 2011; Junjie \& Dingwei, 2006), reflecting the effectiveness of the proposed methodology.

\section{Table 1}

Comparison of results of the algorithms MCBGA, ACO and MGA

\begin{tabular}{|c|c|c|c|c|c|c|c|c|c|c|c|c|c|c|}
\hline \multirow{2}{*}{ Instance } & \multirow{2}{*}{$n$} & \multirow{2}{*}{$\mathrm{C}_{\max }$} & \multicolumn{4}{|c|}{ MCBGA } & \multicolumn{4}{|c|}{$\mathrm{ACO}$} & \multicolumn{4}{|c|}{ MGA } \\
\hline & & & Best & Avg & $\mathrm{T}[\mathrm{s}]$ & $m$ & Best & Avg & $\mathrm{T}[\mathrm{s}]$ & $m$ & Best & Avg & $\mathrm{T}[\mathrm{s}]$ & $m$ \\
\hline pr76 & 76 & 20 & 153774 & 157666,6 & 1,8 & 4 & 178597 & 180690 & 19 & 5 & 157444 & 160574 & 43 & 5 \\
\hline pr152 & 152 & 40 & 119938 & 128768,8 & 11,07 & 4 & 130953 & 136341 & 41 & 5 & 127839 & 133337 & 91 & 5 \\
\hline pr226 & 226 & 50 & 157239 & 160836,4 & 17,63 & 5 & 167646 & 170877 & 62 & 5 & 166827 & 178501 & 195 & 5 \\
\hline pr299 & 299 & 70 & 71081 & 73192,8 & 35,65 & 5 & 82106 & 83845 & 65 & 5 & 82176 & 85796 & 363 & 5 \\
\hline pr436 & 436 & 100 & 136809 & 140436,6 & 80 & 5 & 161955 & 165035 & 95 & 5 & 173839 & 183698 & 623 & 5 \\
\hline pr1002 & 1002 & 220 & 313561 & 318778,8 & 465,7 & 5 & 382198 & 387205 & 186 & 5 & 427269 & 459179 & 2892 & 5 \\
\hline
\end{tabular}

The new best solutions found are presented below:

\section{Instance pr76: 4 routes, cost 153774.}

23222446454448476968706750495354424327 26;

21255556575859604161626364737271656651 52;

283334403938183736353229303119205 4;

2367891012111716151314747576.

\section{Instance pr152: 4 routes, cost 119938.}

354950767475735178465253727170695445445556686766655743425859646362 61604115216 ;

31441351261171089171840192120222339242526272838293031323337 34;

79114117140139141142143138137118119136135144145146134133120121132131147 1481491301291221231281271511501521261251247748 ;

4792939495116115969197989990801131008981101102103888211211110487105106 1071101091088584868336. 
Instance pr226: 5 routes, cost 157239.

74757677787980818283848586111112113114115116117118119120121122123124125 13213013112912712812611010910810710610410510370696871 72;

212211210209208207206205204203202201200199198197196195194193192191190189 18818718618518418318218118017917817717617517417317217114314214814914764 67 13;

87959689889798909199100929310110294137144146145138139140141150170169 16816716616516416316216116015915815715615515415315215141434746 49;

9101415181920212223242526272930343537383940424445364833323128505152 535455565758596061626365171612 8;

234567116613613513413321321421521621721821922022122222322422522673.

\section{Instance pr299: 5 routes, cost 71081.}

378890899186949796959883849910010282817980767877757273747169706768 105646510762636633342928272625242321181716151312111098562 ;

192022303132353638374240414345464447484950515253545655116114115117119 118121122120180181182183184185256186253252255261260254257258259262264263 266265267268248249244247243237236134136 92;

148587101103168167197240242196193192191194195170169174172128109108106110 615960395758112111113123127126124125175177176178187189179171173190188250 251245246270269271239241238272273274235234198164163162 139;

4142141140161138137135133132129104130131165166201199200202228203229231 230233232277276275279278281282283280284285286219221222209220224223226225 227299206205204207208210211160159158157144145 146;

92142212211217215216286287288290289291292294293296295297214213155154153 152151150149148146147.

\section{Instance pr436: 5 routes, cost 136809.}

46789101112131415161732313029282726642523212224656667201918686970 71728889909110410510610714414611211175616059587980818256554847444345 46;

494286224223233234235236237238239240241242243244245246247248249250210211 212213214215216217218219220221191184192183193194195196197198199200201202 203205206172173174204175176177178179180181182154155156157158159160161162 16313413513613713813914014114215215315115014310874736362333435363740 5; 3977761091131141161181191211221011201171151031029293941009596979899125 123124126127129128130131132133167165164166169168170171207208209251253252 255254316315317318319320321322323324325326327328329331332330303302304305 306307308309310311312313314256257258259260261262263264265266267268269270 271;

4138577887110147148145185190222272301273274275276277278279294295296297 298299300333334335336337338339360370361362363364365366367368369378385386 387388401400402404405417418428429431416415419420421414406407413412423422 432433430427424426425410411409408403434438437436435439284344343342281230 1875450 ;

5153189188225231232280285286287340288289290291292293359357358356355354 352353351350349347348346345376377379389390380374375381391392399398396397 3933953943843823833723713733412822292832282272261861498584835223. 


\section{Instance pr1002: 5 routes, cost 313561.}

75779390929198979695136135134133100101102128129130131132137138139140141 142152151150149148144143145146147126127125124123122121257258259256255254 253249250247248260261262246245244243251252995153154155156164165166169168 167163162158157185159160161172171170173174175176177178179180181184183182 996203204206205207210209208200199198201202196197194195187186241242240238 239188189193192190191237236235233234221219218217212211213216215214220222 223224225226227228229230232231263264265266267268269271270272273274275276 277278279280281282301302303304113112114115116118106105994107108109307308 3093103113124544434241505153525473 71;

74808384484746306305991297298286287288403402408409411412413414415417416 410407406405421422420418419426425424423464463462461460458457456432433446 444443442440438439441632631630629611610609608607606605604603602601599597 596595594593592591559560558557556555554553552551550548542543544541539540 545546547549562561563564565519518520521517516567566568570569514515523522 525524530529526527528536537538535533534532531999510511512509508507506505 504503502501498499500489488487513485486490491495496497494493492484483482 481998580579571572573574590589588576575578577581582583584585449448450451 45245345545447547447347147247647747847948047046946846712011711910410399 9476 ;

79788987110111283284428429430431997434447445795796797798799800804805806 809810811812820821822825824823819818814813841815816817828827826829830831 8328338348358368378408398381002859860862861863866865864856855854857858 852853850851843842897898896894895844845849848846847893892891889890877875 874873868867869872871870876878879880881882883884885886888887919900901902 918917916904903906905909910911912915914913777776778779780781783782803802 801787794793788786785784758757756789790791792751752753754755747746748750 749733734735738744745743742741740739737736730731732616615614613612627628 626625992633634635636637638639640641373372371370369377376375374437436435 4274048688 ;

82853002992854664654595865875986008088071001908907899920921922923924925 927926928929930931932933934935936937938939957958959960769767766768770771 7727737757747627617607591000763764765702703704705697698699700701969968 967966965964963962961993953954956955940941942943944945946947948952951949 950977978979976973974975980982981972971970987986985983984990989988684683 685686687682676675677674671670669672673678679681680688689690691692693694 695696706707709708710713711712723722721715714716717718720719668667666665 664663726725724728729727620619618617624623621622649650651648645646647652 654653644643642659658657655656662661660356355357358359354348347349346343 34232731349 81;

341344345350351353352360361362363364365366367368378379381380382385383384 395394393387386388389390392391340339338337336335398397396400401399292291 290289296295293294321322323320317318319324326325316315314331330329328334 33333228272930312620192118151413161722232524323334353637383940585755 567270696867666559606162646312111098764352.

\section{Conclusion}

In this paper, a genetic algorithm modified to solve the mTSP has been presented. In order to examine the efficiency of the algorithm, tests on six instances of literature ranging from 76 to 1002 cities were 
made. The algorithm found better solutions for all instances used with respect to the values reported by Seidighpour et al. (2011) and Junjie and Dingwei (2006). Similarly, the average solutions after 10 run of the algorithm, for each instance, are less than the average reported solutions. The results show that the efficiency of the proposed algorithm, which combines technique initialization and neighborhood structures, provide good quality solutions at each iteration of the algorithm.

\section{References}

Angel, R. D., Caudle, W. L., Noonan, R., \& Whinston, A. N. D. A. (1972). Computer-assisted school bus scheduling. Management Science, 18(6), B-279.

Bektas, T. (2006). The multiple traveling salesman problem: an overview of formulations and solution procedures. Omega, 34(3), 209-219.

Bellmore, M., \& Hong, S. (1974). Transformation of multisalesman problem to the standard traveling salesman problem. Journal of the ACM (JACM), 21(3), 500-504.

Brumitt, B. L., \& Stentz, A. (1998, May). GRAMMPS: A generalized mission planner for multiple mobile robots in unstructured environments. In Robotics and Automation, 1998. Proceedings. 1998 IEEE International Conference on(Vol. 2, pp. 1564-1571). IEEE.

Calvo, R. W., \& Cordone, R. (2003). A heuristic approach to the overnight security service problem. Computers \& Operations Research, 30(9), 1269-1287.

Carter, A. E., \& Ragsdale, C. T. (2002). Scheduling pre-printed newspaper advertising inserts using genetic algorithms. Omega, 30(6), 415-421.

Chen, S. H., \& Chen, M. C. (2011, November). Operators of the two-part encoding genetic algorithm in solving the multiple traveling salesmen problem. In Technologies and Applications of Artificial Intelligence (TAAI), 2011 International Conference on (pp. 331-336). IEEE.

Christofides, N. (1976). Worst-case analysis of a new heuristic for the travelling salesman problem (No. RR-388). Carnegie-Mellon Univ Pittsburgh Pa Management Sciences Research Group.

Chu, P. C., \& Beasley, J. E. (1997). A genetic algorithm for the generalised assignment problem. Computers \& Operations Research, 24(1), 17-23.

Faigl, J., Kulich, M., \& Preucil, L. (2005, September). Multiple traveling salesmen problem with hierarchy of cities in inspection task with limited visibility. In proceedings of the 5th Workshop on Self-Organizing Maps. Universit Paris-Sud (pp. 91-98).

Gilbert, K. C., \& Hofstra, R. B. (1992). A new multiperiod multiple traveling salesman problem with heuristic and application to a scheduling problem.Decision Sciences, 23(1), 250-259.

Goldberg, D. E., \& Deb, K. A comparison of selection scheme used in genetic algorithms. Foundations of Genatic Algorithms, 69-93.

Goldberg, D. E., \& Lingle, R. (1985, July). Alleles, loci, and the traveling salesman problem. In Proceedings of the first international conference on genetic algorithms and their applications (pp. 154-159). Lawrence Erlbaum Associates, Publishers.

Gorenstein, S. (1970). Printing press scheduling for multi-edition periodicals.Management Science, 16(6), B-373.

GuoXing, Y. (1995). Transformation of multidepot multisalesmen problem to the standard travelling salesman problem. European Journal of Operational Research, 81(3), 557-560.

Gutin, G., Yeo, A., \& Zverovich, A. (2002). Traveling salesman should not be greedy: domination analysis of greedy-type heuristics for the TSP. Discrete Applied Mathematics, 117(1), 81-86.

Junjie, P., \& Dingwei, W. (2006, August). An ant colony optimization algorithm for multiple travelling salesman problem. In Innovative Computing, Information and Control, 2006. ICICIC'06. First International Conference on (Vol. 1, pp. 210-213). IEEE.

Laporte, G., \& Nobert, Y. (1980). A cutting planes algorithm for the m-salesmen problem. Journal of the Operational Research Society, 1017-1023.

Lin, S., \& Kernighan, B. W. (1973). An effective heuristic algorithm for the traveling-salesman problem. Operations research, 21(2), 498-516. 
Miller, C. E., Tucker, A. W., \& Zemlin, R. A. (1960). Integer programming formulation of traveling salesman problems. Journal of the ACM (JACM), 7(4), 326-329.

Okonjo-Adigwe, C. (1988). An effective method of balancing the workload amongst salesmen. Omega, 16(2), 159-163.

Oliver, I. M., Smith, D., \& Holland, J. R. (1987). Study of permutation crossover operators on the traveling salesman problem. In Genetic algorithms and their applications: proceedings of the second International Conference on Genetic Algorithms: July 28-31, 1987 at the Massachusetts Institute of Technology, Cambridge, MA. Hillsdale, NJ: L. Erlhaum Associates, $1987 .$.

Reinhelt, G. (2014). \{TSPLIB\}: a library of sample instances for the TSP (and related problems) from various sources and of various types. URL: http://comopt. ifi. uniheidelberg. de/software/TSPLIB95.

Russell, R. A. (1977). Technical Note-An Effective Heuristic for the M-Tour Traveling Salesman Problem with Some Side Conditions. Operations Research, 25(3), 517-524.

Sariel-Talay, S., Balch, T. R., \& Erdogan, N. (2009). Multiple traveling robot problem: A solution based on dynamic task selection and robust execution.Mechatronics, IEEE/ASME Transactions on, 14(2), 198-206.

Sedighpour, M., Yousefikhoshbakht, M., \& Mahmoodi Darani, N. (2012). An Effective Genetic Algorithm for Solving the Multiple Traveling Salesman Problem. Journal of Optimization in Industrial Engineering, (8), 73-79.

Song, C. H., Lee, K., \& Lee, W. D. (2003, July). Extended simulated annealing for augmented TSP and multi-salesmen TSP. In Neural Networks, 2003. Proceedings of the International Joint Conference on (Vol. 3, pp. 2340-2343). IEEE.

Subramanian, A., Penna, P. H. V., Uchoa, E., \& Ochi, L. S. (2012). A hybrid algorithm for the heterogeneous fleet vehicle routing problem. European Journal of Operational Research, 221(2), 285-295.

Svestka, J. A., \& Huckfeldt, V. E. (1973). Computational experience with an m-salesman traveling salesman algorithm. Management Science, 19(7), 790-799.

Syswerda, G. (1991). Schedule optimization using genetic algorithms.Handbook of genetic algorithms.

Sze, S., \& Tiong, W. (2007). A comparison between heuristic and meta-heuristic methods for solving the multiple traveling salesman problem. World Academy of Science, Engineering and Technology, 1.

Tang, L., Liu, J., Rong, A., \& Yang, Z. (2000). A multiple traveling salesman problem model for hot rolling scheduling in Shanghai Baoshan Iron \& Steel Complex. European Journal of Operational Research, 124(2), 267-282.

Yousefikhoshbakht, M., \& Sedighpour, M. (2012). A combination of sweep algorithm and elite ant colony optimization for solving the multiple traveling salesman problem. Proceedings of the Romanian Academy A, 13(4), 295-302.

Yu, Z., Jinhai, L., Guochang, G., Rubo, Z., \& Haiyan, Y. (2002). An implementation of evolutionary computation for path planning of cooperative mobile robots. In Intelligent Control and Automation, 2002. Proceedings of the 4th World Congress on (Vol. 3, pp. 1798-1802). IEEE.

Zhang, T., Gruver, W., \& Smith, M. H. (1999). Team scheduling by genetic search. In Intelligent Processing and Manufacturing of Materials, 1999. IPMM'99. Proceedings of the Second International Conference on (Vol. 2, pp. 839-844). IEEE. 